\title{
Vibrational relaxation of highly excited $\mathrm{HO}_{2}$ in collisions with $\mathrm{O}_{2}$
}

\author{
A.J.C. Varandas *, L. Zhang \\ Departamento de Química, Universidade de Coimbra, 3004-535 Coimbra, Portugal
}

Received 12 November 2004; in final form 17 December 2004

Available online 5 January 2005

\begin{abstract}
We investigate the relaxation of highly vibrationally excited $\mathrm{HO}_{2}$ in collisions with vibrationally cold $\mathrm{O}_{2}$. The calculations employ the quasiclassical trajectory method and a global double many-body expansion potential energy surface for $\mathrm{HO}_{4}\left({ }^{2} \mathrm{~A}\right)$. Both deactivation and activation processes are observed, with the rate constants being found to be strongly influenced by the donor internal energy content. It is also found that the title process may not be ignorable when modelling the ozone budget in the middle atmosphere.
\end{abstract}

(c) 2004 Elsevier B.V. All rights reserved.

\section{Introduction}

Vibrationally excited molecules play a crucial role in many areas of reaction kinetics and dynamics, being largely for this reason that the topic has been and remains a subject of great interest. In particular, collisional relaxation of highly excited molecules is a primary mechanism by which hot molecular systems return to thermal equilibrium. Thus, knowledge of the vibrational deactivation and activation of such molecules is vital to gain insight into their chemical and physical properties. In fact, to understand activated gas phase environments in which the populations of numerous species and states are often controlled by complex kinetic networks, one must know the extent to which vibrationally excited molecules resist collisional relaxation [1,2]. It is therefore not surprising that the last decades have seen an explosive growth of interest in detailed studies of collisional processes involving vibrationally hot systems [3-11].

The hydroperoxyl radical is, in both the ground and vibrationally excited states, of particular interest in the context of the present work because it is a key interme-

\footnotetext{
${ }^{*}$ Corresponding author. Fax: +239 835867.

E-mail address: varandas@qtvs1.qui.uc.pt (A.J.C. Varandas).
}

diate in a number of systems. It plays a prominent role in many reactions such as those involved in atmospheric chemistry [12-19], stratospheric formation and destruction of ozone [20,21], and photochemical air pollution [22-25], with numerous experimental and theoretical investigations having been reported. Although, significant progress has been made in understanding the reaction dynamics of the $\mathrm{HO}_{2}$ radical, complete characterization of the influence of vibrational excitation on the rates of such processes has remained elusive. Recently, a theory has been proposed $[2,26]$ to explain the so-called 'ozone deficit problem' and ' $\mathrm{HO}_{x}$ dilemma' in the middle atmosphere, which suggests that the vibrational relaxation of $\mathrm{HO}_{2}$ may have important implications in modeling the odd-oxygen and odd-hydrogen budgets at such altitudes. An investigation of the dynamics of highly vibrationally excited $\mathrm{HO}_{2}$ in collisions with $\mathrm{O}_{2}$, which is a major constituent of the atmosphere, is therefore an intriguing topic of considerable interest.

In a previous paper [27], we have provided the first information on the $\mathrm{HO}_{2}+\mathrm{O}_{2}$ atmospheric reaction with both reactants being highly vibrationally excited. However, our understanding of the collisional energy transfer behavior of highly excited $\mathrm{HO}_{2}$ radicals (hereafter denoted as $\mathrm{HO}_{2}^{*}$ ) is far from complete since the role of 
vibrational relaxation in such processes has been left unanswered. In fact, collisional deactivation always competes with chemical reaction. Since the loss of energy by $\mathrm{HO}_{2}^{*}$ radicals can play a key role in atmospheric modelling, the need then exists for identifying important physical parameters and propensities in the title quenching process.

Our major interest in this work is twofold: (a) provide a detailed theoretical investigation of the title vibrational relaxation process by considering $\mathrm{HO}_{2}^{*}$ radicals containing between 21 and $48 \mathrm{kcal} \mathrm{mol}^{-1}$ of vibrational excitation and $\mathrm{O}_{2}$ molecules in their ground vibrational state; (b) test further our recently proposed DMBE potential energy surface for ground-doublet $\mathrm{HO}_{4}$ [28], which has been found useful to rationalize the important $\mathrm{OH}(\mathrm{v})+\mathrm{O}_{3}$ atmospheric reaction and its reverse [28$31,27]$. For $\mathrm{OH}(\mathrm{v})+\mathrm{O}_{3}$, not only good agreement with the available experimental data has been obtained, but also the long-standing problem on the $\mathrm{OH}(\mathrm{v})$ removal reaction mechanism involving competition between vibrational relaxation and chemical reaction dynamics has been elucidated. To achieve the above targets, we will follow previous work by employing the well established quasiclassical trajectory (QCT) method for the dynamics calculations. Such a method is not only about the only viable means available for such a study but also justifiable due to the high energies involved. The Letter is organized as follows. Section 2 provides a brief survey of the methodology. The results are presented and discussed in Section 3, and the conclusions gathered in Section 4.

\section{Computational details}

All calculations employed the QCT method as implemented in an extensively adapted version of the MERCURy/Venus 96 [32] codes. They have been done for diatom-triatom translational energies over the range $1 \leqslant E_{\mathrm{tr}} / \mathrm{kcal} \mathrm{mol}^{-1} \leqslant 20$, as summarized in Tables 1 and 2 . The initial conditions were defined by using the standard fixed normal mode sampling procedure which is implemented as an option of such codes. In all cases the initial rotational quantum number of the colliding $\mathrm{O}_{2}$ has been fixed at the ground state level $(j=1)$.

Table 1

Summary of the trajectory calculations for $\mathrm{O}_{2}+\mathrm{HO}_{2}\left(E_{\mathrm{v}}\right)$ deactivation processes

\begin{tabular}{|c|c|c|c|c|c|c|c|}
\hline \multicolumn{4}{|c|}{$E_{\mathrm{V}_{i}}=21 \mathrm{kcal} \mathrm{mol}^{-1}$} & \multicolumn{2}{|c|}{$E_{\mathrm{v}_{i}}=36 \mathrm{kcal} \mathrm{mol}^{-1}$} & \multicolumn{2}{|c|}{$E_{\mathrm{v}_{i}}=48 \mathrm{kcal} \mathrm{mol}^{-1}$} \\
\hline$\overline{\Delta E_{\downarrow}}$ & $E_{\mathrm{tr}}$ & $b_{\max }$ & $\sigma^{\Delta E_{\downarrow}} \pm \Delta \sigma^{\Delta E_{\downarrow}}$ & $b_{\max }$ & $\sigma^{\Delta E_{\downarrow}} \pm \Delta \sigma^{\Delta E_{\downarrow}}$ & $b_{\max }$ & $\sigma^{\Delta E_{\downarrow}} \pm \Delta \sigma^{\Delta E_{\downarrow}}$ \\
\hline \multirow[t]{6}{*}{-1} & 1.0 & 10.39 & $55.15 \pm 2.80$ & 10.77 & $60.87 \pm 3.04$ & 10.96 & $64.16 \pm 3.17$ \\
\hline & 2.0 & 9.83 & $48.54 \pm 2.49$ & 10.20 & $51.69 \pm 2.67$ & 10.39 & $55.66 \pm 2.81$ \\
\hline & 4.0 & 9.26 & $43.10 \pm 2.21$ & 9.64 & $46.40 \pm 2.39$ & 9.83 & $52.33 \pm 2.56$ \\
\hline & 6.0 & 8.50 & $38.73 \pm 1.91$ & 8.88 & $42.13 \pm 2.08$ & 9.07 & $45.75 \pm 2.21$ \\
\hline & 8.0 & 8.13 & $35.26 \pm 1.74$ & 8.50 & $36.35 \pm 1.86$ & 8.69 & $39.17 \pm 1.97$ \\
\hline & 12.0 & 7.75 & $30.17 \pm 1.55$ & 8.13 & $31.74 \pm 1.67$ & 8.31 & $34.43 \pm 1.77$ \\
\hline \multirow[t]{6}{*}{-2} & 1.0 & 10.39 & $23.92 \pm 1.94$ & 10.77 & $28.43 \pm 2.19$ & 10.96 & $38.50 \pm 2.55$ \\
\hline & 2.0 & 9.83 & $21.84 \pm 1.75$ & 10.20 & $24.54 \pm 1.93$ & 10.39 & $33.26 \pm 2.26$ \\
\hline & 4.0 & 9.26 & $20.07 \pm 1.58$ & 9.64 & $21.01 \pm 1.69$ & 9.83 & $30.34 \pm 2.03$ \\
\hline & 6.0 & 8.50 & $18.29 \pm 1.38$ & 8.88 & $18.46 \pm 1.46$ & 9.07 & $25.46 \pm 1.72$ \\
\hline & 8.0 & 8.13 & $16.39 \pm 1.25$ & 8.50 & $15.22 \pm 1.27$ & 8.69 & $22.91 \pm 1.57$ \\
\hline & 12.0 & 7.75 & $13.20 \pm 1.08$ & 8.13 & $12.45 \pm 1.10$ & 8.31 & $19.01 \pm 1.37$ \\
\hline \multirow[t]{6}{*}{-4} & 1.0 & 10.39 & $16.63 \pm 1.64$ & 10.77 & $17.13 \pm 1.73$ & 10.96 & $21.51 \pm 1.96$ \\
\hline & 2.0 & 9.83 & $13.65 \pm 1.41$ & 10.20 & $14.72 \pm 1.52$ & 10.39 & $16.63 \pm 1.64$ \\
\hline & 4.0 & 9.26 & $11.72 \pm 1.23$ & 9.64 & $11.67 \pm 1.28$ & 9.83 & $15.17 \pm 1.48$ \\
\hline & 6.0 & 8.50 & $7.72 \pm 0.92$ & 8.88 & $9.54 \pm 1.07$ & 9.07 & $12.02 \pm 1.22$ \\
\hline & 8.0 & 8.13 & $5.91 \pm 0.77$ & 8.50 & $7.04 \pm 0.88$ & 8.69 & $10.09 \pm 1.07$ \\
\hline & 12.0 & 7.75 & $4.15 \pm 0.62$ & 8.13 & $5.39 \pm 0.74$ & 8.31 & $8.25 \pm 0.93$ \\
\hline \multirow[t]{6}{*}{-6} & 1.0 & 10.39 & $11.37 \pm 1.37$ & 10.77 & $10.21 \pm 1.34$ & 10.96 & $13.59 \pm 1.57$ \\
\hline & 2.0 & 9.83 & $8.95 \pm 1.15$ & 10.20 & $8.67 \pm 1.17$ & 10.39 & $12.22 \pm 1.41$ \\
\hline & 4.0 & 9.26 & $6.60 \pm 0.93$ & 9.64 & $7.15 \pm 1.01$ & 9.83 & $10.31 \pm 1.23$ \\
\hline & 6.0 & 8.50 & $3.29 \pm 0.61$ & 8.88 & $6.20 \pm 0.87$ & 9.07 & $8.40 \pm 1.02$ \\
\hline & 8.0 & 8.13 & $1.87 \pm 0.44$ & 8.50 & $5.79 \pm 0.80$ & 8.69 & $6.77 \pm 0.88$ \\
\hline & 12.0 & 7.75 & $1.70 \pm 0.40$ & 8.13 & $3.63 \pm 0.61$ & 8.31 & $5.10 \pm 0.74$ \\
\hline \multirow[t]{6}{*}{-10} & 1.0 & 10.39 & $2.04 \pm 0.59$ & 10.77 & $3.10 \pm 0.75$ & 10.96 & $4.34 \pm 0.90$ \\
\hline & 2.0 & 9.83 & $1.52 \pm 0.48$ & 10.20 & $2.78 \pm 0.67$ & 10.39 & $3.73 \pm 0.79$ \\
\hline & 4.0 & 9.26 & $0.94 \pm 0.36$ & 9.64 & $2.04 \pm 0.54$ & 9.83 & $3.19 \pm 0.69$ \\
\hline & 6.0 & 8.50 & $0.34 \pm 0.20$ & 8.88 & $1.36 \pm 0.41$ & 9.07 & $2.84 \pm 0.60$ \\
\hline & 8.0 & 8.13 & $0.10 \pm 0.10$ & 8.50 & $1.14 \pm 0.36$ & 8.69 & $2.61 \pm 0.55$ \\
\hline & 12.0 & 7.75 & & 8.13 & $0.83 \pm 0.29$ & 8.31 & $1.96 \pm 0.46$ \\
\hline
\end{tabular}

Energies are in $\mathrm{kcal} \mathrm{mol}^{-1}$, impact parameters in $a_{0}$, and cross-sections in $a_{0}^{2}$. 
Table 2

Summary of the trajectory calculations for $\mathrm{O}_{2}+\mathrm{HO}_{2}\left(E_{\mathrm{v}}\right)$ activation processes

\begin{tabular}{|c|c|c|c|c|c|c|c|}
\hline \multicolumn{4}{|c|}{$E_{\mathrm{v}_{i}}=21 \mathrm{kcal} \mathrm{mol}^{-1}$} & \multicolumn{2}{|c|}{$E_{\mathrm{v}_{i}}=36 \mathrm{kcal} \mathrm{mol}^{-1}$} & \multicolumn{2}{|c|}{$E_{\mathrm{V}_{i}}=48 \mathrm{kcal} \mathrm{mol}^{-1}$} \\
\hline$\Delta E_{\uparrow}$ & $E_{\mathrm{tr}}$ & $b_{\max }$ & $\sigma^{\Delta E_{\uparrow}} \pm \Delta \sigma^{\Delta E_{\uparrow}}$ & $b_{\max }$ & $\sigma^{\Delta E_{\uparrow}} \pm \Delta \sigma^{\Delta E_{\uparrow}}$ & $b_{\max }$ & $\sigma^{\Delta E_{\uparrow}} \pm \Delta \sigma^{\Delta E_{\uparrow}}$ \\
\hline \multirow[t]{6}{*}{1} & 1.0 & 10.39 & $6.28 \pm 1.02$ & 10.77 & $7.29 \pm 1.14$ & 10.96 & $7.55 \pm 1.18$ \\
\hline & 2.0 & 9.83 & $7.58 \pm 1.06$ & 10.20 & $8.83 \pm 1.19$ & 10.39 & $8.99 \pm 1.22$ \\
\hline & 4.0 & 9.26 & $11.85 \pm 1.24$ & 9.64 & $13.27 \pm 1.36$ & 9.83 & $13.80 \pm 1.41$ \\
\hline & 6.0 & 8.50 & $14.20 \pm 1.23$ & 8.88 & $15.86 \pm 1.36$ & 9.07 & $17.45 \pm 1.45$ \\
\hline & 8.0 & 8.13 & $17.42 \pm 1.29$ & 8.50 & $18.63 \pm 1.39$ & 8.69 & $20.89 \pm 1.50$ \\
\hline & 12.0 & 7.75 & $19.14 \pm 1.27$ & 8.13 & $21.16 \pm 1.40$ & 8.31 & $22.26 \pm 1.47$ \\
\hline \multirow[t]{6}{*}{2} & 1.0 & 10.39 & $1.70 \pm 0.54$ & 10.77 & $0.73 \pm 0.36$ & 10.96 & $0.38 \pm 0.27$ \\
\hline & 2.0 & 9.83 & $2.27 \pm 0.59$ & 10.20 & $1.15 \pm 0.43$ & 10.39 & $1.70 \pm 0.54$ \\
\hline & 4.0 & 9.26 & $3.63 \pm 0.70$ & 9.64 & $3.36 \pm 0.70$ & 9.83 & $2.73 \pm 0.64$ \\
\hline & 6.0 & 8.50 & $5.91 \pm 0.81$ & 8.88 & $5.82 \pm 0.84$ & 9.07 & $5.17 \pm 0.81$ \\
\hline & 8.0 & 8.13 & $6.95 \pm 0.83$ & 8.50 & $7.84 \pm 0.93$ & 8.69 & $6.29 \pm 0.85$ \\
\hline & 12.0 & 7.75 & $7.83 \pm 0.84$ & 8.13 & $7.78 \pm 0.88$ & 8.31 & $8.91 \pm 0.89$ \\
\hline \multirow[t]{6}{*}{3} & 1.0 & 10.39 & $0.17 \pm 0.17$ & 10.77 & & 10.96 & $0.19 \pm 0.19$ \\
\hline & 2.0 & 9.83 & $0.46 \pm 0.26$ & 10.20 & $0.32 \pm 0.23$ & 10.39 & $0.68 \pm 0.34$ \\
\hline & 4.0 & 9.26 & $1.35 \pm 0.42$ & 9.64 & $1.75 \pm 0.50$ & 9.83 & $1.21 \pm 0.43$ \\
\hline & 6.0 & 8.50 & $1.70 \pm 0.44$ & 8.88 & $2.73 \pm 0.58$ & 9.07 & $1.94 \pm 0.50$ \\
\hline & 8.0 & 8.13 & $2.18 \pm 0.47$ & 8.50 & $4.43 \pm 0.70$ & 8.69 & $2.97 \pm 0.59$ \\
\hline & 12.0 & 7.75 & $2.83 \pm 0.51$ & 8.13 & $5.08 \pm 0.71$ & 8.31 & $4.23 \pm 0.67$ \\
\hline
\end{tabular}

Energies are in $\mathrm{kcal} \mathrm{mol}^{-1}$, impact parameters in $a_{0}$, and cross-sections in $a_{0}^{2}$.

Although, a more rigorous kinetics treatment would involve an average over all initial rotational states, such a mammoth task is probably unjustified on the basis of previous work [27,31]. Thus, we omit heretofore the specification of the initial rotational state. $E_{\mathrm{v}}=\sum_{k=1}^{3} E_{\mathrm{v}_{k}}$ is the total vibrational energy of $\mathrm{HO}_{2}^{*}$, with $E_{\mathrm{v}_{k}}$ indicating the energy content of each vibrational normal mode (for completeness, we indicate here the fundamental vibrational frequencies of $\mathrm{HO}_{2}$ : $1101 \mathrm{~cm}^{-1}$ (O-O stretching), $1353 \mathrm{~cm}^{-1}$ for (HOO bending), and $3484 \mathrm{~cm}^{-1}\left(\mathrm{H}-\mathrm{O}_{2}\right.$ stretching), corresponding to a zero-point energy of $\left.8.490 \mathrm{kcal} \mathrm{mol}^{-1}\right)$. Note that the chosen range of vibrational excitations for $\mathrm{HO}_{2}\left(E_{\mathrm{v}}\right)$ mimics roughly the one observed in the products of the $\mathrm{OH}(\mathrm{v} \leqslant 4)+\mathrm{O}_{3}$ reaction [30]. The rotational energy about each principal axis of inertia of $\mathrm{HO}_{2}^{*}$ has been taken as $k_{\mathrm{B}} T / 2$, and the rotational temperature assumed to be $300 \mathrm{~K}$. This corresponds to an intermediate value for the range $100 \leqslant T / \mathrm{K} \leqslant 500$, which covers the temperatures of major interest in atmospheric chemistry. The initial diatom-triatom separation has been fixed at $17 a_{0}$ where the interaction energy is essentially negligible, while the optimum step size for integration of the equations of motion has been set according to the usual procedure. In turn, the maximum value of the impact parameter $\left(b_{\max }\right)$ leading to vibrational relaxation has been optimized from batches of 200 trajectories ensuring that the standard deviation between the vibrational energy of the products in the non-reactive trajectories and the vibrational energy of reactants reaches a similar value as for the total energy conservation (this is typically of $0.01 \mathrm{kcal} \mathrm{mol}^{-1}$ ). Batches of 2000 trajectories have then been run for each value of the translational energy $\left(E_{\mathrm{tr}}\right)$ and initial vibrational combination making a total of $3.6 \times 10^{4}$ trajectories.

The energetics of the studied process is best seen from the diagram of Fig. 1, which indicates by the line segments on the reactants side the various vibrational combinations according to the $\mathrm{HO}_{4} \mathrm{DMBE}$ potential energy surface [28]. Note that the internal energy combination $\left(E_{\mathrm{v}_{i}}=48, v=0\right)$ corresponds roughly to the threshold energy for ozone formation. However, for combinations involving lower internal energies, there is not necessarily enough energy to surmount such a barrier. Since all reactive channels are essentially closed over the complete range of translational energies, our work thence focuses on non-reactive regimes.

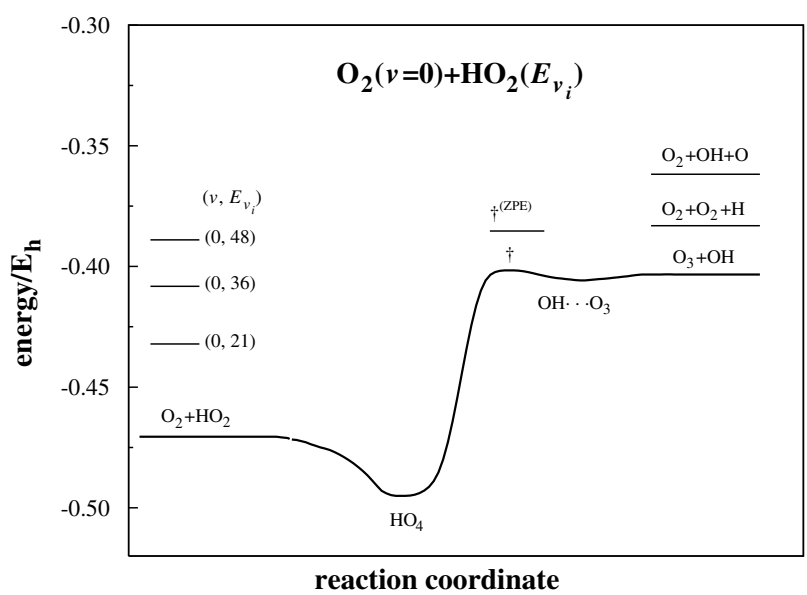

Fig. 1. Schematic diagram showing the energetics of different processes according to the $\mathrm{HO}_{4}$ DMBE potential energy surface. 
In order to describe the state-to-state energy transfer arising from the title relaxation process, we employ the following scheme. For a specific initial vibrational excitation of $\mathrm{HO}_{2}^{*}, E_{v_{i}}$, we count the trajectory outcomes according to their vibrational energy content. First, we have denoted by $\Delta E \mathrm{kcal} \mathrm{mol}^{-1}$ the amount of transferred vibrational energy. Thus, a negative value of $\Delta E$ implies the loss of energy by $\mathrm{HO}_{2}^{*}$ in a deactivation process, while a positive one refers to energy gain via an activation mechanism. Second, we have defined the state-bin as encompassing the range $\left(E_{\mathrm{v}_{i}}+\Delta E\right)-0.5$ $\rightarrow\left(E_{\mathrm{v}_{i}}+\Delta E\right)+0.5$, and considered as vibrationally inelastic trajectories for losing/gaining $\Delta E \mathrm{kcal} \mathrm{mol}^{-1}$ all those whose vibrational energy fell inside such a bin. Needless to say, the above boxing scheme suffers from the usual errors inherent to the partition of the internal energy [32]. This has been tentatively overcome by leaving an interval of $1 \mathrm{kcal} \mathrm{mol}^{-1}$ around the starting energy to account for such errors.

For a given value of the translational energy, the deactivation cross-section is given by $\sigma^{\Delta E_{\downarrow}}=\pi b_{\max }^{2} P^{\Delta E_{\downarrow}}$, with the associated $68 \%$ uncertainties given by $\Delta \sigma^{\Delta E_{\downarrow}}=$ $\left(\frac{N-N^{\Delta E_{\downarrow}}}{N N^{\Delta E_{\downarrow}}}\right)^{1 / 2} \sigma^{\Delta E_{\downarrow}}$; thus, $N^{\Delta E_{\downarrow}}$ is the number of trajectories that lead upon de-excitation to an energy transfer of up to $\Delta E \mathrm{kcal} \mathrm{mol}^{-1}$ out of a total of $N$ collisions starting with an initial vibrational energy of $E_{\mathrm{v}_{i}}$, and $P^{\Delta E_{\downarrow}}=N^{\Delta E_{\downarrow}} / N$ the corresponding vibrational deexcitation probability. From the deactivation cross-section and assuming a Maxwell-Boltzmann distribution over the translational energy, the state-to-state thermal rate coefficients assume the form

$$
\begin{aligned}
k^{\Delta E_{\downarrow}}(T)= & g_{\mathrm{e}}(T)\left(\frac{2}{k_{\mathrm{B}} T}\right)^{3 / 2}\left(\frac{1}{\pi \mu}\right)^{1 / 2} \int_{0}^{\infty} E_{\mathrm{tr}} \sigma^{\Delta E_{\downarrow}} \\
& \times \exp \left(-\frac{E_{\mathrm{tr}}}{k_{\mathrm{B}} T}\right) \mathrm{d} E_{\mathrm{tr}},
\end{aligned}
$$

where $g_{\mathrm{e}}(T)=1 / 3$ is the electronic degeneracy factor (ratio of the electronic partition functions), $k_{\mathrm{B}}$ is the Boltzmann constant, $\mu$ is the reduced mass of the colliding particles, and $T$ is the temperature. Analogous expressions are defined for the activation process.

\section{Results and discussion}

Tables 1 and 2 provide a summary of the trajectory calculations carried out in the present work (for convenience, all data is reported with two decimal figures). Note that the maximum impact parameter slightly increases with internal energy of the reactants for a fixed translational energy. Conversely, $b_{\max }$ is found to increase with decreasing translational energy. Such a dependence on the translational energy is well described by the form $b_{\max }=a E_{\mathrm{tr}}^{-m}$, where $a$ and $m$ are least squares parameters (their numerical values are omitted

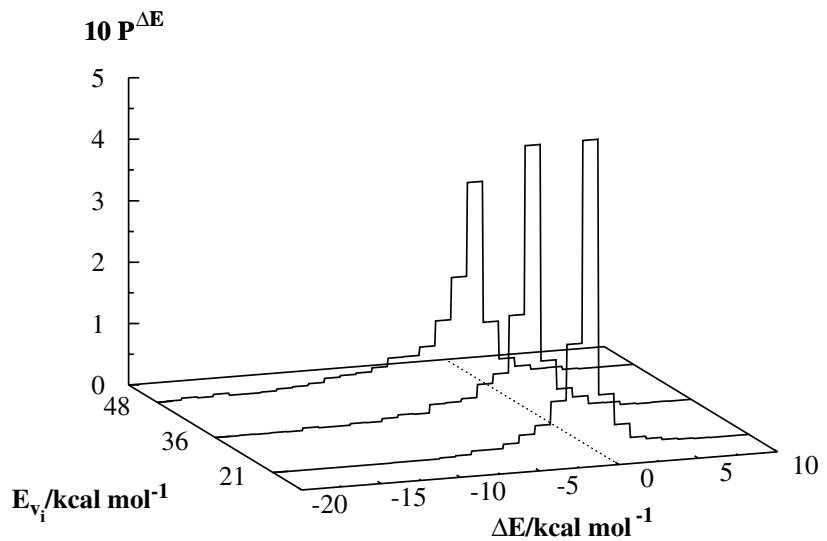

Fig. 2. Vibrational state-to-state energy transfer probability distributions.

for brevity). This may be rationalized from the fact that the de-excitation process involves the coupling of the various degrees of freedom, which is likely to be induced by the long range interactions.

Fig. 2 shows the vibrational state-to-state energy transfer probability distributions for $\mathrm{HO}_{2}^{*}$ with initial vibrational energies of 21,36 , and $48 \mathrm{kcal} \mathrm{mol}^{-1}$. For simplicity, only the case corresponding to $E_{\mathrm{tr}}=8.0$ kcal mol ${ }^{-1}$ is shown in Fig. 2. The notable feature is the fact that both deactivation and activation processes involve frequently multiquantum transitions. Such a mechanism may be justified on the basis of reasonably strong long range interactions, and invoking that an extensive energy exchange between the vibrational modes is required. Also interesting is the fact that such events obey roughly an exponential energy-gap law, a propensity in agreement with other vibrational relaxation processes $[2,11,33-35]$. We further observe that, for a fixed translational energy, the shape of the energy-transfer distribution does not significantly change with the initial vibrational energy, while the amplitude of the multiquantum-transitions in deactivation increases with the internal energy content of the donor species. Thus, one expects more states to be populated upon deactivation.

Table 3 summarizes the information on the products energy partitioning for the deactivation process of $\mathrm{HO}_{2}^{*}$ with $E_{\mathrm{v}_{i}}=48 \mathrm{kcal} \mathrm{mol}^{-1}$. Three points are noteworthy. First, the average value of the vibrational energy in the acceptor $\mathrm{O}_{2}$ molecule is never found to exceed that of the first vibrationally excited level. This implies that a considerable part of the transferred internal energy has been channeled into rotational and translational degrees of freedom. Thus, one expects a small probability for vibration-vibration (V-V) energy transfer to occur (in this case the hot molecule would lose vibrational energy for the acceptor species which would become vibrationally excited). As a result, a vibration to rotation/ translation (V-R, V-T) energy transfer mechanism 
Table 3

Energies (in kcal mol${ }^{-1}$ ) partitioned to different degrees of freedom in the $\mathrm{O}_{2}+\mathrm{HO}_{2}\left(E_{\mathrm{v}}=48\right)$ deactivation process

\begin{tabular}{|c|c|c|c|c|c|c|}
\hline \multirow[t]{2}{*}{$\overline{\Delta E_{\downarrow}}$} & \multirow[t]{2}{*}{$E_{\mathrm{tr}}$} & \multirow[t]{2}{*}{$\left\langle E_{\mathrm{tr}}\right\rangle$} & \multicolumn{2}{|l|}{$\mathrm{HO}_{2}$} & \multicolumn{2}{|l|}{$\mathrm{O}_{2}$} \\
\hline & & & $\left\langle E_{\mathrm{v}}\right\rangle$ & $\left\langle E_{\mathrm{r}}\right\rangle$ & $\left\langle E_{\mathrm{v}}\right\rangle$ & $\left\langle E_{\mathrm{r}}\right\rangle$ \\
\hline \multirow[t]{6}{*}{-1} & 1.0 & 0.90 & 47.06 & 1.49 & 2.24 & 0.23 \\
\hline & 2.0 & 1.59 & 47.03 & 1.65 & 2.25 & 0.30 \\
\hline & 4.0 & 3.00 & 47.05 & 1.84 & 2.26 & 0.48 \\
\hline & 6.0 & 4.40 & 47.02 & 2.18 & 2.24 & 0.64 \\
\hline & 8.0 & 5.84 & 47.05 & 2.35 & 2.35 & 0.76 \\
\hline & 12.0 & 8.99 & 47.06 & 2.74 & 2.34 & 0.85 \\
\hline \multirow[t]{6}{*}{-2} & 1.0 & 1.25 & 46.01 & 2.12 & 2.17 & 0.41 \\
\hline & 2.0 & 1.79 & 46.01 & 2.18 & 2.36 & 0.55 \\
\hline & 4.0 & 3.24 & 46.07 & 2.49 & 2.29 & 0.61 \\
\hline & 6.0 & 4.38 & 46.03 & 2.79 & 2.30 & 1.13 \\
\hline & 8.0 & 5.97 & 46.05 & 3.10 & 2.31 & 1.01 \\
\hline & 12.0 & 9.00 & 45.98 & 3.73 & 2.44 & 1.08 \\
\hline \multirow{6}{*}{-4} & 1.0 & 2.19 & 44.02 & 2.64 & 2.29 & 0.86 \\
\hline & 2.0 & 2.74 & 43.99 & 2.65 & 2.68 & 0.86 \\
\hline & 4.0 & 4.29 & 44.02 & 3.09 & 2.42 & 1.02 \\
\hline & 6.0 & 4.59 & 44.05 & 4.34 & 2.57 & 1.19 \\
\hline & 8.0 & 6.80 & 43.99 & 4.00 & 2.44 & 1.36 \\
\hline & 12.0 & 9.04 & 44.02 & 4.70 & 2.58 & 2.03 \\
\hline \multirow[t]{6}{*}{-6} & 1.0 & 3.39 & 42.04 & 2.93 & 2.49 & 1.17 \\
\hline & 2.0 & 3.97 & 42.05 & 2.94 & 2.55 & 1.44 \\
\hline & 4.0 & 4.76 & 42.00 & 3.72 & 2.73 & 1.63 \\
\hline & 6.0 & 5.32 & 42.01 & 4.25 & 3.13 & 2.06 \\
\hline & 8.0 & 6.82 & 42.00 & 5.36 & 2.86 & 1.68 \\
\hline & 12.0 & 9.62 & 42.06 & 6.32 & 2.82 & 1.77 \\
\hline \multirow[t]{6}{*}{-10} & 1.0 & 4.93 & 38.00 & 3.21 & 3.88 & 1.98 \\
\hline & 2.0 & 5.07 & 38.08 & 4.88 & 3.32 & 1.62 \\
\hline & 4.0 & 6.29 & 38.04 & 5.14 & 3.71 & 1.69 \\
\hline & 6.0 & 8.66 & 38.01 & 5.39 & 2.74 & 1.99 \\
\hline & 8.0 & 9.46 & 37.94 & 6.14 & 2.22 & 2.88 \\
\hline & 12.0 & 11.28 & 38.03 & 7.13 & 2.53 & 3.67 \\
\hline
\end{tabular}

seems to be responsible for the bulk of the deactivation processes here reported. Of course, in classical mechanics one cannot exclude that some vibrational energy will flow into rotation prior to close contact of the two interacting species, although contact may be considered to occur whenever the electronic densities of the two molecules start to overlap each other. Second, for $\Delta E_{\downarrow} \geqslant-2 \mathrm{kcal} \mathrm{mol}^{-1}$, the $\mathrm{V}-\mathrm{R}$ mechanism is found to dominate in the deactivation processes. However, for $\Delta E_{\downarrow}<-2 \mathrm{kcal} \mathrm{mol}^{-1}$, the ratios between the (average) final and initial translational energies tends to decrease with increasing initial translational energy. This suggests that the dependence of energy transfer on the relative translational energy shows two different patterns. For low translational energies, the deactivation process is the result of $\mathrm{V}-\mathrm{T}$ and $\mathrm{V}-\mathrm{R}$ energy transfers, while for high translational energies the $\mathrm{V}-\mathrm{R}$ mechanism seems to offer the best route for deactivation. Third, the percent rotational energy of the final $\mathrm{HO}_{2}$ radical increases with increasing translational energy. Thus, the rotational degrees of freedom of the energy-donor triatomic play a significant role in the collisional deactivation process. The results further suggest that deactivation based on the $\mathrm{V}-\mathrm{R}$ energy exchange mechanism occurs primar- ily via collisions which simply rearrange the energy in the $\mathrm{HO}_{2}^{*}$ molecule, converting vibrational energy to rotational energy. Similar considerations apply to the corresponding activation processes. Note that, for the studied relative translational energy regimes, most of the energy transferred in such processes is via $T \rightarrow \mathrm{V}$ energy conversion.

We now examine the shape of the specific state-tostate cross-sections vs. translational energy which is illustrated in Fig. 3 for one of the internal energies considered in the present work: $E_{\mathrm{v}_{i}}=48 \mathrm{kcal} \mathrm{mol}^{-1}$. Note that panel (a) corresponds to the deactivation process, while panel (b) refers to the activation one. Other cases show similar trends, and hence are omitted for brevity. Clearly, two opposite trends are observed that explain their shapes. The deactivation cross-sections show a markedly decreasing pattern with translational energy, suggesting that a capture-like mechanism arising from the strong coupling of the available degrees of freedom governed by the long range interactions dominates over the whole range of translational energies. Such a behavior is quite similar to the reactive case reported elsewhere [27]. Conversely, for the activation process, one observes the common pattern found in reactions that 

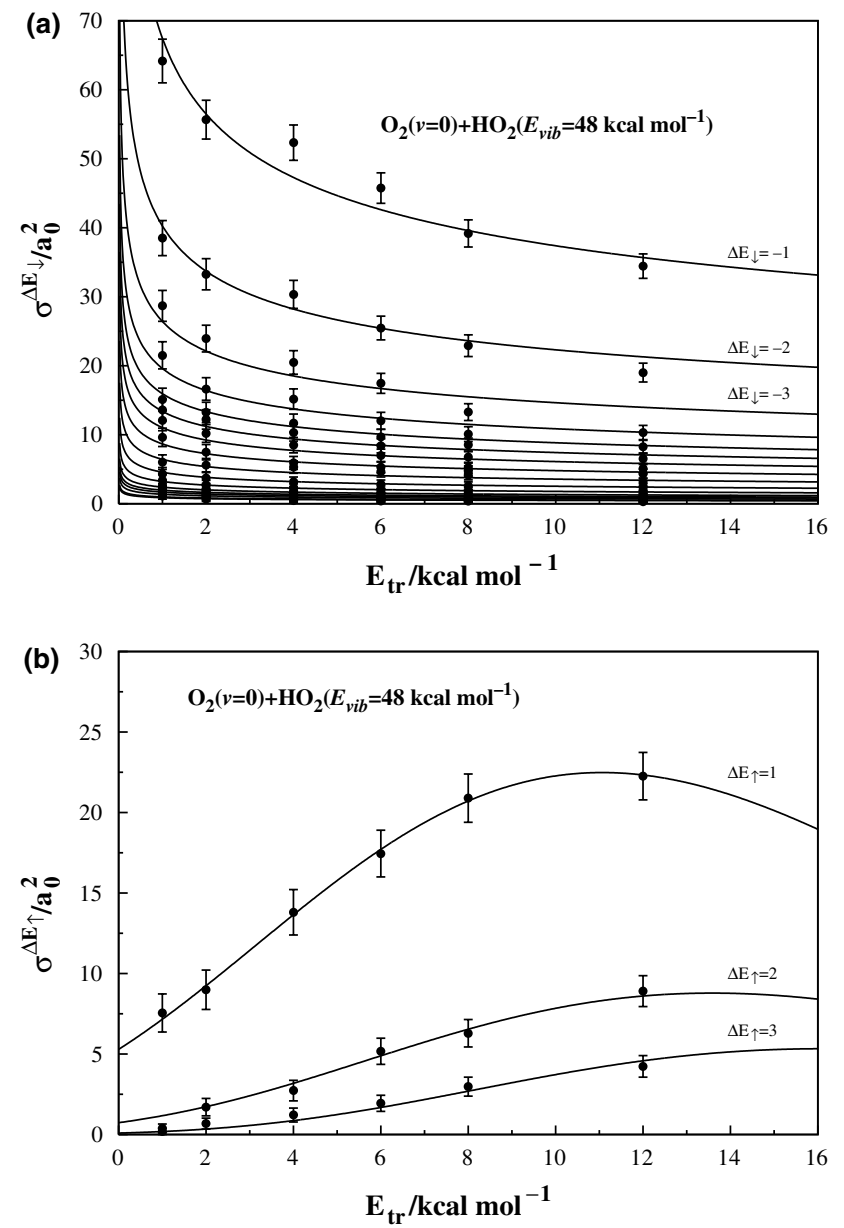

Fig. 3. Cross-sections as a function of the translational energy for $E_{\mathrm{v}_{i}}=48 \mathrm{kcal} \mathrm{mol}^{-1}$ : (a) $\sigma^{\Delta E_{\downarrow}}$; (b) $\sigma^{\Delta E_{\uparrow}}$. Also indicated are the $68 \%$ error bars and the fitted line in Eq. (2).

have an energy threshold: the activation cross-section increases with translational energy until it eventually reaches a maximum and decreases afterwords.

To describe analytically the dependence of the deactivation cross-section with the translational energy we have adopted the following capture-like form:

$\sigma^{\Delta E_{\downarrow}}\left(E_{\mathrm{v}_{i}}, E_{\text {sum }}, E_{\mathrm{tr}}\right)=\frac{\sum_{k=1}^{7} c_{k} E_{\text {sum }}^{k}}{E_{\mathrm{tr}}^{n_{1}} \exp \left[n_{2} E_{\mathrm{v}_{i}}\right]}$,

while the activation part has been written as

$\sigma^{\Delta E_{\uparrow}}\left(E_{\mathrm{V}_{i}}, E_{\mathrm{sum}}, E_{\mathrm{tr}}\right)=\frac{\left[E_{\mathrm{tr}}-E_{\mathrm{tr}}^{\mathrm{th}}\right]^{n_{3}}\left(\sum_{j=0}^{4} d_{j}\left(E_{\mathrm{sum}} / E_{\mathrm{v}_{i}}\right)^{j}\right)}{\exp \left[m\left(E_{\mathrm{tr}}-E_{\mathrm{tr}}^{\mathrm{th}}\right)\right] \exp \left[n_{4} E_{\mathrm{V}_{i}}\right]}$,

where

$E_{\mathrm{tr}}^{\mathrm{th}}=a+b E_{\mathrm{sum}}$

and $E_{\text {sum }}=E_{\mathrm{O}_{2}}+E_{\mathrm{HO}_{2}^{*}}$, with $E_{\mathrm{O}_{2}}$ and $E_{\mathrm{HO}_{2}^{*}}$ being the internal energies of $\mathrm{O}_{2}$ and $\mathrm{HO}_{2}^{*}$. Note that such a rep- resentation depends only on the internal energy of the colliding species, but not on any specific model which expresses the dependence of the internal energy on the quantum numbers. The above model excitation function provides therefore a generalization of that employed elsewhere [29], with the number of parameters and specific algebraic details being chosen to improve the quality of the fit to the calculated data. For accuracy requirements, we first fit separately the calculated results for each of the three distinct initial vibrational energies of $\mathrm{HO}_{2}^{*}$. All parameters have been determined from a least-squares fitting procedure, with their optimum numerical values being reported in columns 2-4 of Table 4 (although, some of the parameters are rather small, we leave their values for reproducibility). The resulting fitted functions are exhibited together with the calculated points in Fig. 3. Clearly, the fitted curves show a good agreement with the calculated points, thus reflecting the general trends of the calculations. Despite its usefulness, such a fit will be unable to predict the cross-sections for other values of $E_{\mathrm{v}_{i}}$ not specifically considered in the fitting procedure. Two other schemes have then been tried. The simplest, model I, consisted of gathering all calculated information into a single least-squares fitting procedure to Eqs. (2)-(4). It turns out that the proposed fitting form is unable to describe well the $E_{\mathrm{v}_{i}}=21 \mathrm{kcal} \mathrm{mol}^{-1}$ case. To account for this, we have then carried out a fit in two steps (model II). First, we have fitted only the points referring to the top curve for each of the three initial vibrational energies studied in the present work. The second step involved a fit to all the remaining calculated points. Model II seems to reasonably predict the cross-sections for initial vibrational energies not actually considered in the present work. To test this assertion, we have compared the predicted and actually computed values for the case where $\mathrm{HO}_{2}^{*}$ has an initial vibrational energy of $42 \mathrm{kcal} \mathrm{mol}^{-1}$. Unfortunately, for space limitations, such a comparison will not be included in the present work. Of course, care must be exercized when using model II to extrapolate far beyond the range of $E_{\mathrm{v}_{i}}$ and $E_{\mathrm{tr}}$ values considered in the fit. Needless to say, neither model I or II are free from some ambiguity, which makes the search for an improved model await future developments.

By substitution of Eqs. (2)-(4) in Eq. (1) and performing the integration analytically, one obtains for the specific state-to-state deactivation and activation rate constants

$$
\begin{aligned}
k^{\Delta E_{\downarrow}}(T)= & g_{\mathrm{e}}(T)\left(\frac{8}{\pi \mu}\right)^{1 / 2}\left(\frac{\sum_{i=1}^{7} c_{i} E_{\text {sum }}^{i}}{\exp \left[n_{2} E_{\mathrm{v}_{i}}\right]}\right) \Gamma\left(2-n_{1}\right) \\
& \times(R T)^{-n_{1}+1 / 2}
\end{aligned}
$$


Table 4

Numerical values of least-squares parameters in Eq. (2)

\begin{tabular}{|c|c|c|c|c|c|c|}
\hline Parameter & $E_{\mathrm{v}_{i}}=21 \mathrm{kcal} \mathrm{mol}^{-1}$ & $E_{\mathrm{v}_{i}}=36 \mathrm{kcal} \mathrm{mol}^{-1}$ & $E_{\mathrm{v}_{i}}=48 \mathrm{kcal} \mathrm{mol}^{-1}$ & Model I & Model II (top line) & Model II (all others) \\
\hline$c_{1}$ & $7.6810(-2)$ & $3.6309(-2)$ & $2.4121(-2)$ & $3.0335(6)$ & 4.3718 & $9.1930(4)$ \\
\hline$c_{2}$ & $-2.6164(-2)$ & $-7.1693(-3)$ & $-3.6424(-3)$ & $-8.7853(5)$ & $1.3786(-2)$ & $-2.6495(4)$ \\
\hline$c_{3}$ & $3.7004(-3)$ & $5.8768(-4)$ & $2.2847(-3)$ & $1.0403(5)$ & $-4.0327(-4)$ & $3.1221(3)$ \\
\hline$c_{4}$ & $-2.7817(-4)$ & $-2.5599(-5)$ & $-7.6195(-6)$ & $-6.4428(3)$ & $-1.9544(-6)$ & $-1.9257(2)$ \\
\hline$c_{5}$ & $1.1723(-5)$ & $6.2494(-7)$ & $1.4248(-7)$ & $2.1997(2)$ & $-1.3017(-8)$ & 6.5620 \\
\hline$c_{6}$ & $-2.6258(-7)$ & $-8.1077(-9)$ & $-1.4165(-9)$ & -3.9253 & $1.3052(-9)$ & -0.1173 \\
\hline$c_{7}$ & $2.4423(-9)$ & $4.3672(-11)$ & $5.8495(-12)$ & $2.8642(-2)$ & $5.7132(-11)$ & $8.6242(-4)$ \\
\hline$n_{1}$ & 0.2570 & 0.2671 & 0.2563 & 0.2628 & 0.2323 & 0.3157 \\
\hline$n_{2}$ & -0.5232 & -0.3663 & -0.2675 & 0.3326 & $2.9017(-2)$ & 0.2667 \\
\hline$d_{0}$ & $1.6900(1)$ & $7.8480(3)$ & $9.2164(3)$ & 2.7016 & 2.7016 & 2.7016 \\
\hline$d_{1}$ & -5.4692 & $-9.2231(3)$ & $-9.5783(3)$ & -8.9084 & -8.9084 & -8.9084 \\
\hline$d_{2}$ & $-1.0311(1)$ & $-6.3173(3)$ & $-8.2966(3)$ & $1.1008(1)$ & $1.1008(1)$ & $1.1008(1)$ \\
\hline$d_{3}$ & -4.7592 & $1.1386(4)$ & $1.1570(4)$ & -6.0417 & -6.0417 & -6.0417 \\
\hline$d_{4}$ & 6.3578 & $-3.5438(3)$ & $-2.7530(3)$ & 1.2426 & 1.2426 & 1.2426 \\
\hline$n_{3}$ & 3.4835 & 1.6997 & 8.9478 & 7.3808 & 7.3808 & 7.3808 \\
\hline$n_{4}$ & 0.1395 & 0.0763 & 0.4001 & 0.0912 & 0.0912 & 0.0912 \\
\hline$m$ & 0.1902 & 0.1077 & 0.3772 & 0.3215 & 0.3215 & 0.3215 \\
\hline$a$ & $-3.6459(1)$ & $-7.8835(1)$ & $-1.4393(1)$ & $-1.1707(1)$ & $-1.1707(1)$ & $-1.1707(1)$ \\
\hline$b$ & 1.2057 & 1.8941 & 2.5172 & $1.3637(-2)$ & $1.3637(-2)$ & $1.3637(-2)$ \\
\hline
\end{tabular}

Units are such that with the energy in $\mathrm{kcal} \mathrm{mol}^{-1}$, the cross-section comes in $a_{0}^{2}$.

and

$$
\begin{aligned}
k^{\Delta E_{\uparrow}}(T)= & g_{\mathrm{e}}(T)\left(\frac{8}{\pi \mu}\right)^{1 / 2}\left\{\left(\sum_{j=0}^{4} d_{j}\left(E_{\mathrm{sum}} / E_{\mathrm{v}_{i}}\right)^{j}\right)\right. \\
& \times \frac{(R T)^{n_{3}+1 / 2} \exp \left[-E_{\mathrm{tr}}^{\mathrm{th}} / R T\right]}{(1+m R T)^{n_{3}+2} \exp \left[n_{4} E_{\mathrm{v}_{i}}\right]}\left[\Gamma\left(n_{3}+2\right)\right. \\
& \left.\left.+\Gamma\left(n_{3}+1\right)(1+m R T) E_{\mathrm{tr}}^{\mathrm{th}} / R T\right]\right\}
\end{aligned}
$$

where $\Gamma(\cdots)$ is the gamma function, and all other symbols have their usual meaning. The perspective view in Fig. 4 shows that the $\mathrm{O}_{2}+\mathrm{HO}_{2}\left(E_{\mathrm{v}_{i}}\right)=48 \mathrm{kcal} \mathrm{mol}^{-1}$ deactivation rate constant increases with temperature while decreasing with de-excitation $\Delta E_{\downarrow}$, with similar considerations applying to the other values of $E_{\mathrm{v}_{i}}$ studied in the present work. We observe that it assumes typical values of some $10^{-11} \mathrm{~cm}^{3} \mathrm{~s}^{-1}$, thus being of the same order of magnitude as the total rate constant for ozone formation in $\mathrm{O}_{2}\left(\mathrm{v}^{\prime}\right)+\mathrm{HO}_{2}\left(E_{\mathrm{V}_{i}}\right)$ reactive collisions [27] which is shown in Table 5 under the heading $R_{\mathrm{v}^{\prime}}(R$ stands for reactive, and $\mathrm{v}^{\prime}$ for the vibrational quantum number of $\mathrm{O}_{2}$; the vibrational energy in $\mathrm{HO}_{2}^{*}$ is uniformly distributed [27] among all normal modes and specified by the value of $\left.E_{\mathrm{v}_{i}}\right)$. In fact, for a given $E_{\mathrm{v}_{i}}$, the $\Delta E_{\downarrow}=-1 \mathrm{kcal} \mathrm{mol}^{-1}$ de-activation rate constants are predicted to be typically a factor of 2 smaller than the reactive rate constants for $R_{27}$, but about 2 times larger than the corresponding $R_{18}$ ones. However, if we consider instead the total relaxation rate constant $k^{\downarrow}(T)=\sum_{\Delta E_{\downarrow}} k^{\Delta E_{\downarrow}}(T)$ also reported in Table 5, the ratio $k^{\downarrow}(T) / R_{\mathrm{v}^{\prime}}(T)$ for $E_{\mathrm{v}_{i}}=36 \mathrm{kcal} \mathrm{mol}^{-1}$ at $T=250 \mathrm{~K}$ attains the values of 6.4 and 1.4 , respectively for $\mathrm{v}^{\prime}=18$ and 27. Correspondingly, for $E_{\mathrm{v}_{i}}=48 \mathrm{kcal} \mathrm{mol}^{-1}$ and

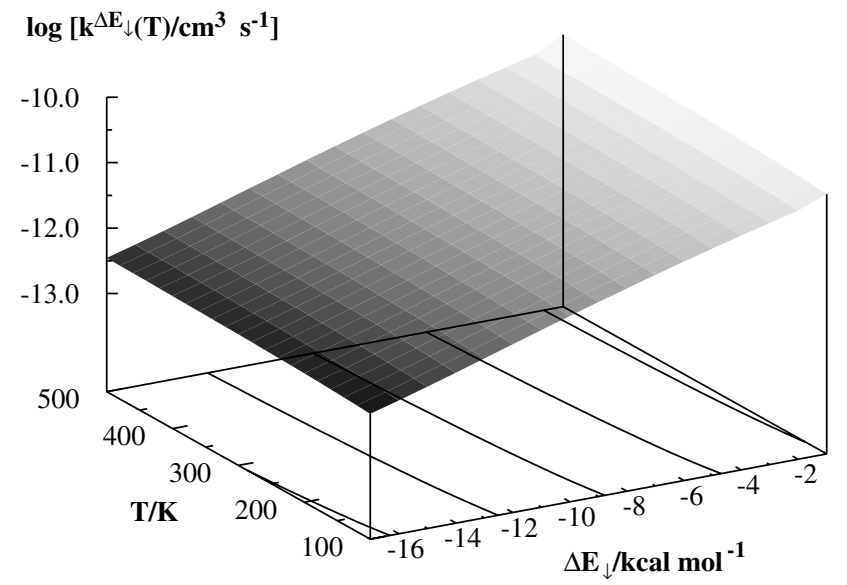

Fig. 4. Thermal de-activation rate coefficients for $\mathrm{O}_{2}+\mathrm{HO}_{2}\left(E_{\mathrm{v}_{i}}\right)$ as a function of temperature; $E_{\mathrm{v}_{i}}=48 \mathrm{kcal} \mathrm{mol}^{-1}$.

$\mathrm{v}^{\prime}=27$ at the same temperature, such a ratio assumes the value of 1.7. Furthermore, model II is seen to give results that differ by only a few percent from those based on the more accurate vibrational-energy-specific excitation functions, and hence the values reported for $E_{\mathrm{v}_{i}}=42 \mathrm{kcal} \mathrm{mol}^{-1}$ should be reliable. Thus, although the rate constant for $\mathrm{O}_{2}\left(\mathrm{v}^{\prime}\right)+\mathrm{HO}_{2}\left(E_{\mathrm{v}}\right)$ reactive scattering can be appreciably large, the relaxation of $\mathrm{HO}_{2}\left(E_{\mathrm{v}}\right)$ in collisions with cold $\mathrm{O}_{2}$ may not be ignorable. Note however that, if the vibrational energy loss is only 1 $\mathrm{kcal} \mathrm{mol}^{-1}$ (this is by far the dominant contribution), it should not be much important from the point of view of reactivity since the molecule would keep basically intact its potential for reacting with a vibrationally hot $\mathrm{O}_{2}$ in a subsequent collision. To clarify the importance of 


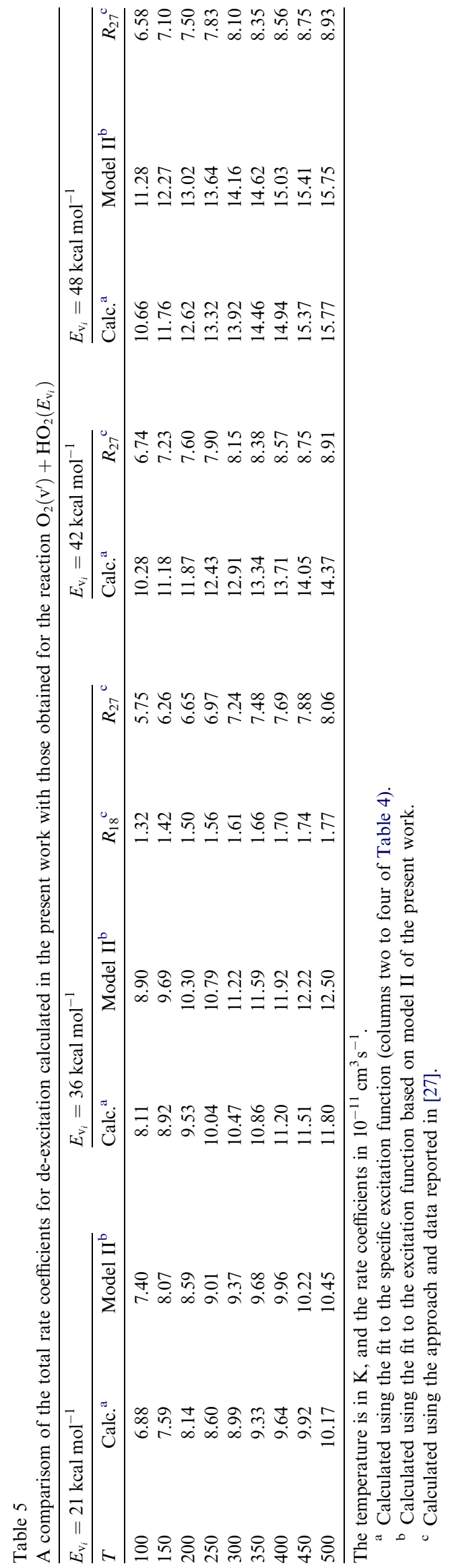

energy transfer at high altitudes, let us consider for simplicity that $k^{\downarrow}(T)=R_{\mathrm{v}^{\prime}}(T)$. The ratio between the rates of reaction with vibrationally hot $\mathrm{O}_{2}$ and vibrational deactivation in collisions with cold $\mathrm{O}_{2}$ is then approximately given by the ratio $\left[\mathrm{O}_{2}\left(\mathrm{v}^{\prime}\right)\right] /\left[\mathrm{O}_{2}\left(\mathrm{v}^{\prime}=0\right)\right]$. Clearly, the importance of the title deactivation processes in the middle atmosphere depends on the fraction of vibrationally hot oxygen molecules: it increases with decreasing $\left[\mathrm{O}_{2}\left(\mathrm{v}^{\prime}\right)\right] /\left[\mathrm{O}_{2}\left(\mathrm{v}^{\prime}=0\right)\right]$. Since this ratio may be appreciable $[11,2]$ at high altitudes and most deactivated molecules may keep their reacting capability nearly intact upon a single collision, it is probably safe to emphasize prior to a deeper kinetics analysis that both non-reactive and reactive processes should be considered when analyzing the ozone budget in the middle atmosphere.

\section{Concluding remarks}

We have reported, to our knowledge, the first theoretical study of energy transfer in collisions of high vibrationally excited hydroperoxyl radicals with ground state molecular oxygen. The calculations predict the relaxation of $\mathrm{HO}_{2}^{*}$ to occur primarily via $\mathrm{V}-\mathrm{R}$ and $\mathrm{V}-$ $\mathrm{T}$ mechanisms. Specifically, the $\mathrm{V}-\mathrm{R}$ mechanism is found to dominate in the deactivation process at high translational energy regimes, while the $\mathrm{V}-\mathrm{V}$ exchange energy process is generally found to play a minor role. The calculations further suggest that the deactivation process is to a large extent governed by the long range interactions as shown by the capture-like shape of the calculated excitation function. Conversely, activation processes depict the usual form of excitation function. As for practical implications, it is concluded that vibrational relaxation may not be ignorable when discussing the ozone budget in the middle atmosphere. Unfortunately, no comparison with experimental data has been possible due to the unavailability of the latter. It is hoped that this work may stimulate experimental efforts along such a direction.

\section{Acknowledgements}

This work has the support of Fundação para a Ciência e Tecnologia (Portugal) within the auspices of the POCTI programme, and of FEDER.

\section{References}

[1] T.J. Bevilacqua, R.B. Weisman, J. Chem. Phys. 98 (1993) 6316.

[2] A.J.C. Varandas, J. Phys. Chem. A 108 (2004) 758.

[3] C.R. Gallucci, G. Schatz, J. Phys. Chem. 86 (1982) 2352.

[4] R.J. Gordon, Comment. At. Mol. Phys. 21 (1988) 123.

[5] R.G. Gilbert, S.C. Smith, Theory of Unimolecular and Recombination Reactions, Blackwell Scientific Publications, Oxford, 1990. 
[6] I. Oref, D.C. Tardy, Chem. Rev. 90 (1990) 1407.

[7] R.E. Weston, G.W. Flynn, Annu. Rev. Phys. Chem. 43 (1992) 559.

[8] M.C. Wall, A.S. Lemoff, A.S. Mullin, J. Phys. Chem. A 102 (1998) 9101

[9] J.D. Garrido, P.J.S.B. Caridade, A.J.C. Varandas, J. Phys. Chem. A 106 (2002) 5314.

[10] P.J.S.B. Caridade, J. Sabin, J.D. Garrido, A.J.C. Varandas, Phys. Chem. Chem. Phys 4 (2002) 4959

[11] A.J.C. Varandas, J. Phys. Chem. A 107 (2003) 3769.

[12] D.R. Bates, M. Nicolet, J. Geophys. Res. 55 (1950) 301.

[13] B.G. Hunt, J. Geophys. Res. 71 (1966) 1385.

[14] M. Nicolet, Planet. Space Sci. 20 (1972) 1671.

[15] F.S. Rowland, M. Molina, Rev. Geophys. Space Phys. 13 (1975) 1.

[16] M. Nicolet, Rev. Geophys. Space Phys. 13 (1975) 593.

[17] F. Kaufman, Annu. Rev. Phys. Chem. 30 (1979) 411.

[18] J.S. Chang, W.H. Duewer, Annu. Rev. Phys. Chem. 30 (1979) 443.

[19] M. Nicolet, Adv. Chem. Phys. 55 (1985) 63.

[20] W.H. Duewer, D.J. Wuebbles, H.W. Ellsaesser, J.S. Chang, J. Geophys. Res. 82 (1977) 935.
[21] P.S. Nangia, S.W. Benson, J. Am. Chem. Soc. 102 (1980) 3105.

[22] T.T. Paukert, H.S. Johnston, J. Chem. Phys. 56 (1972) 2824.

[23] G. Poulet, G.L. Bras, J. Combourieu, J. Chem. Phys. 69 (1978) 767.

[24] R. Atkinson, K.R. Darnall, A.C. Lloyd, A.M. Winer, J.N.J. Pitts, Adv. Photochem. 11 (1979) 375.

[25] T.J. Leck, E.L.J. Cook, J.W. Birks, J. Chem. Phys. 72 (1980) 2364.

[26] A.J.C. Varandas, Chem. Phys. Chem. 3 (2002) 433.

[27] L. Zhang, A.J.C. Varandas, J. Phys. Chem. A 106 (2002) 11911.

[28] A.J.C. Varandas, L. Zhang, Chem. Phys. Lett. 331 (2000) 474.

[29] A.J.C. Varandas, L. Zhang, Chem. Phys. Lett. 340 (2001) 62.

[30] L. Zhang, A.J.C. Varandas, Phys. Chem. Chem. Phys. 3 (2001) 1439.

[31] L. Zhang, A.J.C. Varandas, J. Phys. Chem. A 105 (2001) 10347.

[32] W.L. Hase, Mercury: a general Monte-Carlo classical trajectory computer program, QCPE\#453. An updated version of this code is Venus 96: W.L. Hase, et al. (Ed.), QCPE Bull. 16 (1996) 43.

[33] W. Forst, A.P. Penner, J. Chem. Phys. 72 (1980) 1435.

[34] H. Teitelbaum, Chem. Phys. 124 (1988) 55.

[35] R.A. Bollati, J.C. Ferrero, J. Phys. Chem. 98 (1994) 3933. 